\title{
Optimal partial information control of SPDEs with delay and time-advanced backward SPDEs
}

\author{
Bernt Øksendal* $\quad$ Agnès Sulem ${ }^{\dagger} \quad$ Tusheng Zhang ${ }^{\ddagger}$ \\ 4 May 2011
}

\begin{abstract}
We study optimal control problems for (time-) delayed stochastic partial differential equations with jumps, where the controller has only a partial information flow available. We establish sufficient and necessary (Pontryagin-Bismut-Bensoussan type) maximum principles for an optimal control of such systems. The associated adjoint processes are shown to satisfy a (time-) advanced backward stochastic partial differential equation (ABSPDE). Several results on existence and uniqueness of such ABSPDEs are shown. The results are illustrated by an application to a harvesting problem from a biological system (e.g. a fish population), where the dynamics of the population is modeled by a stochastic reaction-diffusion equation.
\end{abstract}

MSC (2010): 60H15, 93E20, 93C23, 92D25

*Center of Mathematics for Applications (CMA), Dept. of Mathematics, University of Oslo, P.O. Box 1053 Blindern, N-0316 Oslo, Norway, email: oksendal@math.uio.no and Norwegian School of Economics and Business Administration, Helleveien 30, N-5045 Bergen, Norway. The research leading to these results has received funding from the European Research Council under the European Community's Seventh Framework Programme (FP7/2007-2013) / ERC grant agreement no [228087].

${ }^{\dagger}$ INRIA Paris-Rocquencourt, Domaine de Voluceau, Rocquencourt, BP 105, Le Chesnay Cedex, 78153, France, email: agnes.sulem@inria.fr

${ }^{\ddagger}$ School of Mathematics, University of Manchester, Oxford Road, Manchester M139PL, United Kingdom, email:Tusheng.zhang@manchester.ac.uk 
Keywords: Stochastic partial differential equations (SPDEs), delay, optimal control, time-advanced backward SPDEs, optimal harvesting

\section{Introduction}

Let $B(t)=B(t, \omega)$ be a Brownian motion and $\tilde{N}(d t, d \zeta):=N(d t, d \zeta)-$ $\nu(d \zeta) d t$, where $\nu$ is the Lévy measure of the jump measure $N(\cdot, \cdot)$, be an independent compensated Poisson random measure on a filtered probability space $\left(\Omega, \mathcal{F},\left\{\mathcal{F}_{t}\right\}_{0 \leq t \leq T}, P\right)$.

If the density $Y(t, x)$ of a fish population at time $t$ and at the point $x$ is exposed to a harvesting rate density $c(t, x) \geq 0$, the corresponding population state dynamics may be modeled by the following equation:

$$
\begin{aligned}
d Y(t, x) & =\left(\frac{1}{2} \Delta Y(t, x)+\alpha Y(t, x)+\beta Y(t-\delta, x)-c(t, x)\right) d t \\
& +\sigma_{0} Y(t, x) d B(t)+\int_{\mathbb{R}} \gamma_{0}(\zeta) Y\left(t^{-}, x\right) \tilde{N}(d t, d \zeta),
\end{aligned}
$$

where

$$
\Delta=\sum_{i=1}^{n} \frac{\partial^{2}}{\partial x_{i}^{2}}
$$

is the Laplacian operator acting on $x$. Here $\alpha, \beta, \sigma_{0}$ are constants and $\gamma_{0}$ is deterministic.

This is a stochastic partial differential equation (SPDE) of reaction-diffusion type. The Laplacian operator models the diffusion (distribution in space), while the other terms model the local growth at each point $x$. For biological reasons it is natural to include a delay term like $\beta Y(t-\delta, x)$ in the dynamics.

Suppose we want to find a harvesting rate density $c(t, x)$ which maximizes the total expected utility of the harvest plus the utility of the remaining population at a terminal time $T>0$. We assume that at any time the controller (harvester) has only a partial information flow $\mathcal{E}_{t}$ available to base her decision on.

Then the problem is to maximize

$$
J(c)=E\left[\int_{D}\left(\int_{0}^{T} U_{1}(t, x, c(t, x)) d t+U_{2}(x, Y(T, x))\right) d x\right]
$$

over all admissible $\mathcal{E}_{t}$ - predictable harvesting rate densities $c(t, x)$, where $U_{1}, U_{2}$ are given utility functions and $D$ is the region of the lake. 
This is an example of a partial information optimal control problem of an SPDE with delay.

In this paper we prove a sufficient version and a necessary version of a Pontryagin-Bismut-Bensoussan type maximum principle (Sections 2 and 3). These maximum principles involve time-advanced backward SPDEs with jumps for the adjoint processes. In Section 4 we prove existence and uniqueness theorems for such equations. Then in Section 5 we apply these results to study the harvesting problem above.

This paper is close in spirit and methods to the paper [17]. However, there are also essential differences, and the results of the current paper are new.

\section{A sufficient maximum principle}

Let $D$ be a given bounded domain in $\mathbb{R}^{n}$. We consider a general system where the state $Y(t, x)$ at time $t$ and at the point $x \in D \subset \mathbb{R}$ is given by a stochastic partial differential equation (SPDE) with delay, as follows:

$$
\begin{aligned}
d Y(t, x) & =L Y(t, x) d t+b(t, x, Y(t, x), Z(t, x), A(t, x), u(t, x)) d t \\
& +\sigma(t, x, Y(t, x), Z(t, x), A(t, x), u(t, x)) d B(t) \\
& +\int_{\mathbb{R}} \gamma\left(t, x, Y\left(t^{-}, x\right), Z\left(t^{-}, x\right), A\left(t^{-}, x\right), u(t, x), \zeta\right) \tilde{N}(d t, d \zeta) ;(t, x) \in[0, T] \times D
\end{aligned}
$$

with initial values

$$
Y(t, x)=\xi(t, x) ;(t, x) \in[-\delta, 0] \times D
$$

and boundary values

$$
Y(t, x)=0 ;(t, x) \in[0, T] \times \partial D .
$$

Here $L$ is a linear differential operator acting on $x$,

$$
Z(t, x):=Y(t-\delta, x)
$$

is the $\delta$-delay of $Y(t, x)$ and

$$
A(t, x):=\int_{t-\delta}^{t} Y(s, x) d s
$$


is a moving average of delayed values of $Y(t, x)$. We assume that the coefficients

$$
\begin{array}{r}
b(t, x, y, z, a, u):[0, T] \times D \times \mathbb{R} \times \mathbb{R} \times \mathbb{R} \times U \rightarrow R, \\
\sigma(t, x, y, z, a, u):[0, T] \times D \times \mathbb{R} \times \mathbb{R} \times \mathbb{R} \times U \rightarrow R,
\end{array}
$$

and

$$
\gamma(t, x, y, z, a, u, \zeta):[0, T] \times D \times \mathbb{R} \times \mathbb{R} \times \mathbb{R} \times U \times \mathbb{R} \rightarrow R
$$

are $C^{1}$ functions with respect to $y, z, a$ and $u$. The set of possible control values $u(t, x, \omega) \in \mathbb{R}$ is denoted by $U$. The performance functional has the form

$$
J(u)=E\left[\int_{0}^{T} \int_{D} f(t, x, Y(t, x), Z(t, x), A(t, x), u(t, x)) d x d t+\int_{D} h(x, Y(T, x)) d x\right]
$$

where $f:[0, T] \times D \times \mathbb{R}^{4} \rightarrow \mathbb{R}$ and $h:[0, T] \times \mathbb{R} \rightarrow \mathbb{R}$ is a given profit rate and terminal payoff rate, respectively, both assumed to be $C^{1}$ with respect to $y, z, a$ and $u$.

Let $\mathcal{E}_{t} \subseteq \mathcal{F}_{t}$ be a given subfiltration, representing the information available to the controller at time $t$. For example, we could have

$$
\mathcal{E}_{t}=\mathcal{F}_{(t-c)^{+}} ; t \geq 0
$$

for some constant $c>0$, corresponding to a delayed information flow. Let $\mathcal{A}=\mathcal{A}_{\mathcal{E}}$ be a given family of admissible controls, contained in the set of $\mathcal{E}_{t^{-}}$ predictable processes $u(t, x)$ such that (2.1)-(2.3) has a unique solution and $J(u)$ converges absolutely.

Define the Hamiltonian

$$
H:[0, T] \times \mathbb{R}^{n} \times \mathbb{R} \times \mathbb{R} \times \mathbb{R} \times U \times \mathbb{R} \times \mathbb{R} \times \mathcal{R} \rightarrow \mathbb{R}
$$

by

$$
\begin{aligned}
H(t, x, y, z, a, u, p, q, r(\cdot)) & =f(t, x, y, z, a, u)+b(t, x, y, z, a, u) p \\
& +\sigma(t, x, y, z, a, u) q+\int_{\mathbb{R}} \gamma(t, x, y, z, a, u, \zeta) r(\zeta) \nu(d \zeta)
\end{aligned}
$$

where $\mathcal{R}$ is the set of functions $\mathbb{R}_{0} \rightarrow \mathbb{R}$ such that (2.7) converges. To $H$ we associate the following backward SPDE (BSPDE) in the unknown processes 


$$
\begin{aligned}
& p(t, x), q(t, x), r(t, x, \zeta): \\
& d p(t, x)=-g(t, x) d t+q(t, x) d B(t)+\int_{\mathbb{R}} r(t, x, \zeta) \tilde{N}(d t, d \zeta) ;(t, x) \in[0, T] \times D \\
& p(T, x)=\frac{\partial h}{\partial y}(x, Y(T, x)) ; x \in D \\
& p(t, x)=0 ;(t, x) \in(0, T) \times \partial D
\end{aligned}
$$

The driver $g$ in the BSPDE (2.8) is defined by

$$
g(t, x)=E\left[\mu(t, x) \mid \mathcal{F}_{t}\right]
$$

where

$$
\begin{aligned}
\mu(t, x)= & \frac{\partial H}{\partial y}(t, x, Y(t, x), Z(t, x), A(t, x), u(t, x), p(t, x), q(t, x), r(t, x, \cdot)) \\
+ & \frac{\partial H}{\partial z}(t+\delta, x, Y(t+\delta, x), Z(t+\delta, x), A(t+\delta, x), u(t+\delta, x), p(t+\delta, x), \\
& q(t+\delta, x), r(t+\delta, x)) \cdot \chi_{[0, T-\delta]}(t) \\
+ & \int_{t}^{t+\delta} \frac{\partial H}{\partial a}(s, x, Y(s, x), Z(s, x), A(s, x), u(s, x), p(s, x), q(s, x) r(s, x, \cdot)) \chi_{[0, T-\delta]}(s) d s \\
+ & L^{*} p(t, x) .
\end{aligned}
$$

Here $L^{*}$ is the adjoint of $L$, in the sense that $\left(L^{*} \varphi, \psi\right)=(\varphi, L \psi)$; for all $\varphi, \psi \in \mathcal{C}_{0}^{2}\left(\mathbb{R}^{n}\right)$ where $\left(\varphi_{1}, \varphi_{2}\right)=\int_{\mathbb{R}^{n}} \varphi_{1}(x) \varphi_{2}(x) d x$ denotes the inner product in $L^{2}\left(\mathbb{R}^{n}\right)$.

Note that this BSPDE is anticipative, or time-advanced, in the sense that the process $\mu(t, x)$ in the driver contains future values of $X(s, x), u(s, x)$, $p(s, x), q(s, x), r(s, x, \cdot) ; s \leq t+\delta$.

In the SDE case and when there are no jumps and no integral terms in the system, similar anticipative BSDEs (A-BSDEs for short) have been studied by Peng and Yang (2009) [22], who prove existence and uniqueness of such equations under certain conditions. They also relate a class of linear A-BSDEs to a class of linear stochastic delay control problems with no delay in the noise coefficients. Thus, in our paper we extend this relation to general nonlinear, partial information, SPDE control problems and general nonlinear A-BSPDEs by means of the maximum principle, and throughout the discussion we include the possibility of delays also in all the noise coefficients, as well as the possibility of jumps. 
Theorem 2.1 (Sufficient maximum principle) Let $\hat{u} \in \mathcal{A}$ with corresponding solutions $\hat{Y}(t, x), \hat{Z}(t, x), \hat{A}(t, x), \hat{p}(t, x), \hat{q}(t, x)$ and $\hat{r}(t, x, \zeta)$. Assume that

$$
y \rightarrow h(x, y) \text { is concave for all } x
$$

and that

$(y, z, a, u) \rightarrow H(t, x, y, z, a, u, \hat{p}(t, x), \hat{q}(t, x), \hat{r}(t, x, \cdot))$ is concave, for all $t, x$.

Moreover, assume that

$$
\begin{aligned}
& E\left[\int _ { 0 } ^ { T } \int _ { D } \left\{\hat{p}^{2}(t, x)[(\sigma(t, x, Y(t, x), Z(t, x), A(t, x), u(t, x))\right.\right. \\
& -\sigma(t, x, \hat{Y}(t, x), \hat{Z}(t, x), \hat{A}(t, x), \hat{u}(t, x)))^{2} \\
+ & \int_{\mathbb{R}}(\gamma(t, x, Y(t, x), Z(t, x), A(t, x), u(t, x), \zeta) \\
-\quad & \left.\gamma(t, x, \hat{Y}(t, x), \hat{Z}(t, x), \hat{A}(t, x), \hat{u}(t, x), \zeta))^{2} \nu(d \zeta)\right] \\
& +(Y(t, x)-\hat{Y}(t, x))^{2}\left[\hat{q}^{2}(t, x)\right. \\
+ & \left.\left.\left.\int_{\mathbb{R}} \hat{r}(t, x, \zeta)^{2} \nu(d \zeta)\right]\right\} d x d t\right]<\infty
\end{aligned}
$$

for all $u \in \mathcal{A}_{\mathcal{E}}$, and that the following maximum condition holds:

$$
\begin{aligned}
& \max _{v \in U} E {\left[H(t, x, \hat{Y}(t, x), \hat{Z}(t, x), \hat{A}(t, x), v, \hat{p}(t, x), \hat{q}(t, x), \hat{r}(t, x, \cdot)) \mid \mathcal{E}_{t}\right] } \\
&=E\left[H(t, x, \hat{Y}(t, x), \hat{Z}(t, x), \hat{A}(t, x), \hat{u}(t, x), \hat{p}(t, x), \hat{q}(t, x), \hat{r}(t, x, \cdot)) \mid \mathcal{E}_{t}\right] .
\end{aligned}
$$

for a.e. $(t, x, \omega) \in[0, T] \times D \times \Omega$.

Then $\hat{u}(t, x)$ is an optimal control.

Proof of Theorem 2.1 Choose $u \in \mathcal{A}$ and put $Y=Y^{u}$ etc. Then we can write

$$
J(u)-J(\hat{u})=I_{1}+I_{2},
$$

where

$$
\begin{gathered}
I_{1}=E\left[\int_{0}^{T} \int_{D}\{f(t, x, Y(t, x), Z(t, x), A(t, x), u(t, x))]\right. \\
-f(t, x, \hat{Y}(t, x), \hat{Z}(t, x), \hat{A}(t, x), \hat{u}(t, x))\} d x d t]
\end{gathered}
$$


and

$$
I_{2}=E\left[\int_{D}\{h(x, Y(T, x))-h(x, \hat{Y}(T, x))\} d x\right] .
$$

By the definition of $H$ we have

$$
\begin{aligned}
I_{1}= & E\left[\int_{0}^{T} \int_{D}(H(t, x, Y(t, x), Z(t, x), A(t, x), u(t, x), \hat{p}(t, x), \hat{q}(t, x), \hat{r}(t, x, \cdot))\right. \\
& -H(t, x, \hat{Y}(t, x), \hat{Z}(t, x), \hat{A}(t, x), \hat{u}(t, x), \hat{p}(t, x), \hat{q}(t, x), \hat{r}(t, x, \cdot)) \\
& -\{b(t, x, Y(t, x), Z(t, x), A(t, x), u(t, x))-b(t, x, \hat{Y}(t, x), \hat{Z}(t, x), \hat{A}(t, x), \hat{u}(t, x))\} \hat{p}(t, x) \\
& -\{\sigma(t, x, Y(t, x), Z(t, x), A(t, x), u(t, x))-\sigma(t, x, \hat{Y}(t, x), \hat{Z}(t, x), \hat{A}(t, x), \hat{u}(t, x))\} \hat{q}(t, x) \\
& -\int_{\mathbb{R}}\{\gamma(t, x, Y(t, x), Z(t, x), A(t, x), u(t, x), \zeta) \\
& -\gamma(t, x, \hat{Y}(t, x), \hat{Z}(t, x), \hat{A}(t, x), \hat{u}(t, x), \zeta)\} \hat{r}(t, x, \zeta) \nu(d \zeta))] d x d t \\
\leq & E\left[\int _ { 0 } ^ { T } \int _ { D } \left\{\frac{\partial \hat{H}}{\partial y}(t, x)(Y(t, x)-\hat{Y}(t, x))+\frac{\partial \hat{H}}{\partial z}(t, x)(Z(t, x)-\hat{Z}(t, x))\right.\right. \\
& +\frac{\partial \hat{H}}{\partial a}(t, x)(A(t, x)-\hat{A}(t, x))+\frac{\partial \hat{H}}{\partial u}(t, x)(u(t, x)-\hat{u}(t, x)) \\
& -(b(t, x)-\hat{b}(t, x)) \hat{p}(t, x)-(\sigma(t, x)-\hat{\sigma}(t, x)) \hat{q}(t, x) \\
& \left.\left.-\int_{\mathbb{R}}(\gamma(t, x, \zeta)-\hat{\gamma}(t, x, \zeta)) \hat{r}(t, x, \zeta) \nu(d \zeta)\right\} d x d t\right],
\end{aligned}
$$

where we have used the abbreviations

$$
\begin{aligned}
\frac{\partial \hat{H}}{\partial y}(t, x) & =\frac{\partial H}{\partial y}(t, x, \hat{Y}(t, x), \hat{Z}(t, x), \hat{A}(t, x), \hat{u}(t, x)), \\
b(t, x) & =b(t, x, Y(t, x), Z(t, x), A(t, x), u(t, x)), \\
\hat{b}(t, x) & =b(t, x, \hat{Y}(t, x), \hat{Z}(t, x), \hat{A}(t, x), \hat{u}(t, x)), \text { etc. }
\end{aligned}
$$


By concavity of $h$ and the Itô formula we have

$$
\begin{aligned}
I_{2} & \leq E\left[\int_{D} \frac{\partial h}{\partial y}(t, \hat{Y}(T, x))(Y(T, x)-\hat{Y}(T, x)) d x\right] \\
& =E\left[\int_{D} \hat{p}(T, x)(Y(T, x)-\hat{Y}(T, x)) d x\right] \\
& =E\left[\int _ { D } \int _ { 0 } ^ { T } \left\{\left(Y\left(t^{-}, x\right)-\hat{Y}\left(t^{-}, x\right)\right) d \hat{p}(t, x)+\hat{p}\left(t^{-}, x\right) d(Y(t, x)-\hat{Y}(t, x))\right.\right. \\
& \left.\left.+\{\sigma(t, x)-\hat{\sigma}(t, x)\} \hat{q}(t, x)+\int_{\mathbb{R}}\{\gamma(t, x, \zeta)-\hat{\gamma}(t, x, \zeta)\} \hat{r}(t, x, \zeta) \nu(d \zeta)\right\} d t d x\right] .
\end{aligned}
$$

Adding (2.17) and (2.18) we get

$$
\begin{aligned}
J(u)-J(\hat{u}) & \leq E\left[\int _ { D } \int _ { 0 } ^ { T } \left\{\frac{\partial \hat{H}}{\partial y}(t, x)(Y(t, x)-\hat{Y}(t, x))+\frac{\partial \hat{H}}{\partial z}(t, x)(Z(t, x)-\hat{Z}(t, x))\right.\right. \\
& +\frac{\partial \hat{H}}{\partial a}(t, x)(A(t, x)-\hat{A}(t, x))+\frac{\partial \hat{H}}{\partial u}(t, x)(u(t, x)-\hat{u}(t, x)) \\
& -(Y(t, x)-\hat{Y}(t, x)) \hat{g}(t, x)+\hat{p}(t, x)(L Y(t, x)-L \hat{Y}(t, x))\} d x d t] .
\end{aligned}
$$

Note that, since $Y(t, x)=\hat{Y}(t, x)$ for $t \in[-\delta, 0]$ we have

$$
\begin{gathered}
\int_{0}^{T} \frac{\partial \hat{H}}{\partial z}(t, x)(Z(t, x),-\hat{Z}(t, x)) d t=\int_{0}^{T} \frac{\partial \hat{H}}{\partial z}(t, x)(Y(t-\delta, x)-\hat{Y}(t-\delta, x)) d t \\
=\int_{0}^{T} \frac{\partial \hat{H}}{\partial z}(t+\delta, x)(Y(t, x)-\hat{Y}(t, x)) \chi_{[0, T-\delta]}(t) d t
\end{gathered}
$$

Similarly, changing the order of integration gives

$$
\begin{gathered}
\int_{0}^{T} \frac{\partial \hat{H}}{\partial a}(t, x)(A(t, x)-\hat{A}(t, x)) d t=\int_{0}^{T} \frac{\partial \hat{H}}{\partial a}(t, x) \int_{t-\delta}^{t}(Y(s, x)-\hat{Y}(s, x)) d s d t \\
=\int_{0}^{T}\left(\int_{t}^{t+\delta} \frac{\partial \hat{H}}{\partial a}(s, x) d s\right)(Y(t, x)-\hat{Y}(t, x)) \chi_{[0, T-\delta]}(t) d t .
\end{gathered}
$$


Substituting (2.20) and (2.21) into (2.19) we get

$$
\begin{aligned}
J(u)-J(\hat{u}) & \leq E\left[\int _ { D } \int _ { 0 } ^ { T } \left(\left\{\frac{\partial \hat{H}}{\partial y}(t, x)+\frac{\partial \hat{H}}{\partial z}(t+\delta, x) \chi_{[0, T-\delta]}(t)\right.\right.\right. \\
& \left.+\int_{t}^{t+\delta} \frac{\partial \hat{H}}{\partial a}(s, x) d s \chi_{[0, T-\delta]}(t)-\hat{g}(t, x)+L^{*} \hat{p}(t, x)\right\}\{Y(t, x)-\hat{Y}(t, x)\} \\
& \left.\left.+\frac{\partial \hat{H}}{\partial u}(t, x)(u(t, x)-\hat{u}(t, x))\right) d t d x\right]
\end{aligned}
$$

By our definition (2.9) of the driver $g$, this reduces to

$$
\begin{aligned}
J(u)-J(\hat{u}) & \leq E\left[\int_{D} \int_{0}^{T} \frac{\partial \hat{H}}{\partial u}(t, x)(u(t, x)-\hat{u}(t, x)) d t d x\right] \\
& =E\left[\int_{D} \int_{0}^{T} E\left[\frac{\partial \hat{H}}{\partial u}(t, x)(u(t, x)-\hat{u}(t, x)) \mid \mathcal{E}_{t}\right] d t d x\right] \\
& =E\left[\int_{D} \int_{0}^{T} E\left[\frac{\partial \hat{H}}{\partial u}(t, x) \mid \mathcal{E}_{t}\right](u(t, x)-\hat{u}(t, x)) d t d x\right] \leq 0,
\end{aligned}
$$

since $\hat{u}$ maximizes the conditional Hamiltonian.

\section{A necessary maximum principle}

A weakness of the sufficient maximum principle obtained in the previous section is the rather restrictive concavity conditions, which do not always hold in applications. Therefore it is of interest to obtain a maximum principle which does not need these conditions. To this end, we need to make the following assumptions:

A 1 For all $t_{0}, s \in[0, T], t_{0}<s$, and all bounded $\mathcal{E}_{t_{0}}-$ measurable random variables $\alpha$ and all bounded, deterministic functions $\varphi: D \rightarrow \mathbb{R}$ the process $\beta$ defined by

$$
\beta(t, x, \omega)=\alpha(\omega) \chi_{\left[t_{0}, s\right]}(t) \varphi(x) ;(t, x, \omega) \in[0, T] \times D \times \Omega
$$

is in $\mathcal{A}_{\mathcal{E}}$. 
A 2 For all $u \in \mathcal{A}_{\mathcal{E}}$ and all bounded $\beta \in \mathcal{A}_{\mathcal{E}}$ there exists $\delta>0$ such that $u+s \beta \in \mathcal{A}_{\mathcal{E}}$ for all $s \in(-\delta, \delta)$.

A 3 For all $u \in \mathcal{A}$ and bounded $\beta \in \mathcal{A}_{\mathcal{E}}$ the derivative process

$$
\eta(t, x):=\left.\frac{d}{d s} Y^{u+s \beta}(t, x)\right|_{s=0}
$$

exists and belongs to $L^{2}([0, T] \times D \times \Omega)$.

We see that, by (2.1),

$$
\begin{aligned}
d \eta(t, x)= & L \eta(t, x) d t+\left[\frac{\partial b}{\partial y}(t, x) \eta(t, x)+\frac{\partial b}{\partial z}(t, x) \eta(t-\delta, x)\right. \\
& \left.+\frac{\partial b}{\partial a}(t, x) \int_{t-\delta}^{t} \eta(s, x) d s+\frac{\partial b}{\partial u}(t, x) \beta(t, x)\right] d t \\
+ & {\left[\frac{\partial \sigma}{\partial y}(t, x) \eta(t, x)+\frac{\partial \sigma}{\partial z}(t, x) \eta(t-\delta, x)+\frac{\partial \sigma}{\partial a}(t, x) \int_{t-\delta}^{t} \eta(s, x) d s+\frac{\partial \sigma}{\partial u}(t, x) \beta(t, x)\right] d B(t} \\
+ & \int_{\mathbb{R}}\left[\frac{\partial \gamma}{\partial y}(t, x, \zeta) \eta(t, x)+\frac{\partial \gamma}{\partial z}(t, x, \zeta) \eta(t-\delta, x)+\frac{\partial \gamma}{\partial a}(t, x, \zeta) \int_{t-\delta}^{t} \eta(s, x) d s\right. \\
& \left.+\frac{\partial \gamma}{\partial u}(t, x, \zeta) \beta(t, x)\right] \tilde{N}(d t, d \zeta)
\end{aligned}
$$

where we have used the short-hand notation

$$
\frac{\partial b}{\partial y}(t, x)=\frac{d}{d y} b(t, x, y, Z(t), A(t), u(t))_{y=Y(t)} \text { etc. }
$$

Note that

$$
\left.\frac{d}{d s} Z^{u+s \beta}(t, x)\right|_{s=0}=\left.\frac{d}{d s} Y^{u+s \beta}(t-\delta, x)\right|_{s=0}=\eta(t-\delta, x)
$$

and

$$
\left.\frac{d}{d s} A^{u+s \beta}(t, x)\right|_{s=0}=\left.\frac{d}{d s} \int_{t-\delta}^{t} Y^{u+s \beta}(r, x) d r\right|_{s=0}=\int_{t-\delta}^{t} \eta(r, x) d r .
$$

Also, note that

$$
\eta(t, x)=0 \text { for }-\delta \leq t \leq 0 .
$$

We now state and prove the main result of this section: 
Theorem 3.1 (Necessary maximum principle) Suppose $\hat{u} \in \mathcal{A}_{\mathcal{E}}$ with corresponding solutions $\hat{Y}(t, x), \hat{Z}(t, x), \hat{A}(t, x), \hat{\eta}(t, x)$ of $(2.1)-(2.3)$ and (3.1), and adjoint processes $\hat{p}(t, x), \hat{q}(t, x), \hat{r}(t, x, \zeta)$ solving (2.8)-(2.9). Suppose the following growth condition holds:

$$
\begin{aligned}
E & {\left[\int _ { 0 } ^ { T } \int _ { D } \hat { p } ^ { 2 } ( t , x ) \left\{\left(\frac{\partial \sigma}{\partial y}\right)^{2}(t, x) \hat{\eta}^{2}(t, x)+\left(\frac{\partial \sigma}{\partial z}\right)^{2}(t, x) \hat{\eta}^{2}(t-\delta, x)\right.\right.} \\
& +\left(\frac{\partial \sigma}{\partial a}\right)^{2}(t, x)\left(\int_{t-\delta}^{t} \hat{\eta}(t, x) d r\right)^{2}+\left(\frac{\partial \sigma}{\partial u}\right)^{2}(t, x) \\
& +\int_{\mathbb{R}_{0}}\left\{\left(\frac{\partial \theta}{\partial y}\right)^{2}(t, x, \zeta) \hat{\eta}^{2}(t, x)+\left(\frac{\partial \theta}{\partial z}\right)^{2}(t, x, \zeta) \hat{\eta}^{2}(t-\delta, x)\right. \\
& \left.\left.+\left(\frac{\partial \theta}{\partial a}\right)^{2}(t, x, \zeta)\left(\int_{t-\delta}^{t} \hat{\eta}(r, x) d r\right)^{2}+\left(\frac{\partial \theta}{\partial u}\right)^{2}(t, x, \zeta)\right\} \nu(d \zeta)\right\} d x d t \\
& \left.+\int_{0}^{T} \int_{D} \hat{\eta}^{2}(t, x)\left\{\hat{q}^{2}(t, x)+\int_{\mathbb{R}_{0}} \hat{r}^{2}(t, x, \zeta) \nu(d \zeta)\right\} d x d t\right]<\infty
\end{aligned}
$$

Then the following are equivalent:

(i) $\left.\frac{d}{d s} J(\hat{u}+s \beta)\right|_{s=0}=0$ for all bounded $\beta \in \mathcal{A}_{\mathcal{E}}$.

(ii) $E\left[\frac{\partial H}{\partial u}(t, x, \hat{Y}(t, x), \hat{Z}(t, x), \hat{A}(t, x), u, \hat{p}(t, x), \hat{q}(t, x), \hat{r}(t, x, \cdot)) \mid \mathcal{E}_{t}\right]_{u=\hat{u}(t, x)}=$ 0 for all $t \in[0, T]$ and a.a. $x \in D$. 
Proof. $\quad$ (i) $\Rightarrow$ (ii): Assume that (i) holds. For notational simplicity we write $\hat{u}=u, \hat{Y}=Y$, etc... in the following. Then we have

$$
\begin{aligned}
& 0=\left.\frac{d}{d s} J(u+s \beta)\right|_{s=0} \\
& =\frac{d}{d s} E\left[\int_{0}^{T} \int_{D} f\left(t, x, Y^{u+s \beta}(t, x), Z^{u+s \beta}(t, x), A^{u+s \beta}(t, x),(u+s \beta)(t, x)\right) d x d t\right. \\
& \left.+\int_{D} h\left(x, Y^{u+s \beta}(T, x)\right) d x\right] \\
& =E\left[\int _ { 0 } ^ { T } \int _ { D } \left\{\frac{\partial f}{\partial y}(t, x) \eta(t, x)+\frac{\partial f}{\partial z}(t, x) \eta(t-\delta, x)+\frac{\partial f}{\partial a}(t, x) \int_{t-\delta}^{t} \eta(s, x) d s\right.\right. \\
& \left.\left.+\frac{\partial f}{\partial u}(t, x) \beta(t, x)\right\} d x d t+\int_{D} \frac{\partial h}{\partial y}(x, Y(T, x)) \eta(T, x) d x\right] \\
& =E\left[\int _ { 0 } ^ { T } \int _ { D } \left[\left\{\frac{\partial H}{\partial y}(t, x)-p(t, x) \frac{\partial b}{\partial y}(t, x)-q(t, x) \frac{\partial \sigma}{\partial y}(t, x)\right.\right.\right. \\
& \left.-\int_{\mathbb{R}} r(t, x, \zeta) \frac{\partial \gamma}{\partial y}(t, x, \zeta) \nu(d \zeta)\right\} \eta(t, x) \\
& +\left\{\frac{\partial H}{\partial z}(t, x)-p(t, x) \frac{\partial b}{\partial z}(t, x)-q(t, x) \frac{\partial \sigma}{\partial z}(t, x)-\int_{\mathbb{R}} r(t, x, \zeta) \frac{\partial \gamma}{\partial z}(t, x, \zeta) \nu(d \zeta)\right\} \eta(t-\delta, x) \\
& +\left\{\frac{\partial H}{\partial a}(t, x)-p(t, x) \frac{\partial b}{\partial a}(t, x)-q(t, x) \frac{\partial \sigma}{\partial a}(t, x)\right. \\
& \left.-\int_{\mathbb{R}} r(t, x, \zeta) \frac{\partial \gamma}{\partial a}(t, x, \zeta) \nu(d \zeta)\right\} \int_{t-\delta}^{t} \eta(s, x) d s \\
& \left.+\left\{\frac{\partial H}{\partial u}(t, x)-p(t, x) \frac{\partial b}{\partial u}(t, x)-q(t, x) \frac{\partial \sigma}{\partial u}(t, x)-\int_{\mathbb{R}} r(t, x, \zeta) \frac{\partial \gamma}{\partial u}(t, x, \zeta) \nu(d \zeta)\right\} \beta(t, x)\right] d x d t \\
& \left.+\int_{D} \frac{\partial h}{\partial y}(x, Y(T, x)) \eta(T, x) d x\right]
\end{aligned}
$$


By (2.8) and (3.1),

$$
\begin{aligned}
& E\left[\int_{D} \frac{\partial h}{\partial y}(x, Y(t, x)) \eta(T, x) d x\right]=E\left[\int_{D} p(T, x) \eta(T, x) d x\right] \\
& =E\left[\int _ { D } \left\{\int_{0}^{T} p\left(t^{-}, x\right) d \eta(t, x)+\int_{0}^{T} \eta\left(t^{-}, x\right) d p(t, x)\right.\right. \\
& +\int_{0}^{T} q(t, x)\left[\frac{\partial \sigma}{\partial y}(t, x) \eta(t, x)+\frac{\partial \sigma}{\partial z}(t, x) \eta(t-\delta, x)\right. \\
& \left.+\frac{\partial \sigma}{\partial a}(t, x) \int_{t-\delta}^{t} \eta(s, x) d s+\frac{\partial \sigma}{\partial u}(t, x) \beta(t, x)\right] d t \\
& +\int_{0}^{T} \int_{\mathbb{R}} r(t, x, \zeta)\left[\frac{\partial \gamma}{\partial y}(t, x, \zeta) \eta(t, x)+\frac{\partial \gamma}{\partial z}(t, x, \zeta) \eta(t-\delta, x)+\frac{\partial \gamma}{\partial a}(t, x, \zeta) \int_{t-\delta}^{t} \eta(s, x) d s\right. \\
& \left.\left.\left.+\frac{\partial \gamma}{\partial u}(t, x, \zeta) \beta(t, x)\right] \nu(d \zeta) d t\right\} d x\right] \\
& =E\left[\int _ { D } \left\{\int _ { 0 } ^ { T } p ( t ^ { - } , x ) \left[L \eta(t, x)+\left\{\frac{\partial b}{\partial y}(t, x) \eta(t, x)+\frac{\partial b}{\partial z}(t, x) \eta(t-\delta, x)\right.\right.\right.\right. \\
& \left.+\frac{\partial b}{\partial a}(t, x) \int_{t-\delta}^{t} \eta(s, x) d s+\frac{\partial b}{\partial u}(t, x) \beta(t, x)\right] d t \\
& +\int_{0}^{T} \eta\left(t^{-}, x\right)[-g(t, x)] d t \\
& +\int_{0}^{T} q(t, x)\left[\frac{\partial \sigma}{\partial y}(t, x) \eta(t, x)+\frac{\partial \sigma}{\partial z}(t, x) \eta(t-\delta, x)\right. \\
& \left.+\frac{\partial \sigma}{\partial z}(t, x) \int_{t-\delta}^{t} \eta(s, x) d s+\frac{\partial \sigma}{\partial u}(t, x) \beta(t, x)\right] d t \\
& +\int_{0}^{T} \int_{D} r(t, x, \zeta)\left[\frac{\partial \gamma}{\partial y}(t, x, \zeta) \eta(t, x)+\frac{\partial \gamma}{\partial z}(t, x, \zeta) \eta(t-\delta, x)+\frac{\partial \gamma}{\partial a}(t, x, \zeta) \int_{t-\delta}^{t} \eta(s, x) d s\right. \\
& \left.\left.\left.+\frac{\partial \gamma}{\partial u}(t, x, \zeta) \beta(t, x)\right] \nu(d \zeta) d t\right\} d x\right] .
\end{aligned}
$$


Adding (3.2) and (3.3) and using (2.9) we get

$$
\begin{aligned}
0= & E\left[\int _ { D } \left\{\int_{0}^{T} \eta(t, x)\left(\frac{\partial H}{\partial y}(t, x)-g(t, x)\right) d t+\int_{0}^{T} \eta(t-\delta, x) \frac{\partial H}{\partial z}(t, x) d t\right.\right. \\
& \left.\left.+\int_{0}^{T}\left(\int_{t-\delta}^{t} \eta(s, x) d x\right) \frac{\partial H}{\partial a}(t, x) d t+\int_{0}^{T} \frac{\partial H}{\partial u}(t, x) \beta(t, x) d t\right\} d x\right] \\
= & E\left[\int _ { D } \left\{\int _ { 0 } ^ { T } \eta ( t , x ) \left(\frac{\partial H}{\partial y}(t, x)-\frac{\partial H}{\partial y}(t, x)-\frac{\partial H}{\partial z}(t+\delta, x) \chi_{[0, T-\delta]}(t)\right.\right.\right. \\
& \left.-\int_{t}^{t+\delta} \frac{\partial H}{\partial a}(s, x) \chi_{[0, T-\delta]}(s) d s-L^{*} p(t, x)\right) \\
+ & \int_{0}^{T} \eta(t-\delta, x) \frac{\partial H}{\partial z}(t, x) d t+\int_{0}^{T}\left(\int_{t-\delta}^{t} \eta(s, x) d s\right) \frac{\partial H}{\partial a}(t, x) d t \\
+ & \left.\left.\left.\int_{0}^{T} \frac{\partial H}{\partial u}(t, x) \beta(t, x) d t\right\} d x+\int_{0}^{T} p(t, x) L \eta(t, x) d t\right\} d x\right] .
\end{aligned}
$$

Since $Y(t, x)=0$ for $(t, x) \in(0, T) \times \partial D$, we also have $\eta(t, x)=0$ for $(t, x) \in(0, T) \times \partial D$. Moreover, we have $p(t, x)=0$ for $(t, x) \in(0, T) \times \partial D$ by (2.8). Therefore

$$
\int_{D} p(t, x) L \eta(t, x) d x=\int_{D} L^{*} p(t, x) \eta(t, x) d x \text { for } t \in(0, T) .
$$

Note that, by (3.2) and a change of variable,

$$
\begin{gathered}
\int_{0}^{T} \eta(t, x) \frac{\partial H}{\partial z}(t+\delta, x) \chi_{[0, T-\delta]}(t) d t=\int_{\delta}^{T+\delta} \eta(r-\delta, x) \frac{\partial H}{\partial z}(r, x) \chi_{[0, T-\delta]}(r-\delta) d r \\
=\int_{0}^{T} \eta(r-\delta, x) \frac{\partial H}{\partial z}(r, x) d r .
\end{gathered}
$$

Similarly, by changing the order of integration,

$$
\int_{0}^{T} \eta(t, x) \int_{t}^{t+\delta} \frac{\partial H}{\partial a}(s, x) \chi_{[0, T-\delta]}(s) d s d t=\int_{0}^{T} \frac{\partial H}{\partial a}(s, x) \int_{s-\delta}^{s} \eta(t, x) d t d s .
$$

Combining (3.6)-(3.9) we conclude that

$$
E\left[\int_{D} \int_{0}^{T} \frac{\partial H}{\partial u}(t, x) \beta(t, x) d t d x\right]=0 .
$$


Since this holds for all bounded $\beta \in \mathcal{A}_{\mathcal{E}}$ we can for any fixed $0 \leq t_{0} \leq$ $s \leq T$ apply it to

$$
\beta(t, x)=\chi_{\left[t_{0}, s\right]}(t) \alpha(\omega) \varphi(x) ;(t, x, \omega) \in[0, T] \times D \times \Omega,
$$

for all bounded $\mathcal{E}_{t_{0}}$-measurable $\alpha$ and all bounded (deterministic) $\varphi(x)$ in $D$ (see Assumption A1). Then (3.10) becomes

$$
E\left[\int_{D}\left(\int_{t_{0}}^{s} \frac{\partial H}{\partial u}(t, x) d t\right) \varphi(x) d x \alpha\right]=0 .
$$

Differentiating with respect to $s$ we get

$$
E\left[\int_{D} \frac{\partial H}{\partial u}\left(t_{0}, x\right) \varphi(x) d x \alpha\right]=0
$$

Since this holds for all $\alpha$ and $\varphi$ we conclude that

$$
E\left[\frac{\partial H}{\partial u}\left(t_{0}, x\right) \mid \mathcal{E}_{t_{0}}\right]=0 \text { for a.a. }\left(t_{0}, x\right) \in[0, T] \times D,
$$

which is (ii).

(ii) $\Rightarrow$ (i): The proof works both ways: If (ii) holds, then (3.12) holds and hence (3.11) also. Then we obtain (3.10) for all bounded $\beta \in \mathcal{A}$ by taking limits of linear combinations of the $\beta$ 's in Assumption A1. Then the computation above shows that $(3.10) \Rightarrow(\mathrm{i})$.

\section{Time-advanced BSPDEs with jumps}

We now study time-advanced backward stochastic partial differential equations driven both by Brownian motion $B(t)$ and compensated Poisson random measures $\tilde{N}(d t, d z)$.

\subsection{Framework}

Let $B(t)=B(t, \omega)$ be a Brownian motion and $\tilde{N}(d t, d z):=N(d t, d z)-$ $\nu(d z) d t$, where $\nu$ is the Lévy measure of the jump measure $N(\cdot, \cdot)$, be an independent compensated Poisson random measure on a filtered probability 
space $\left(\Omega, \mathcal{F},\left\{\mathcal{F}_{t}\right\}_{0 \leq t \leq T}, P\right)$. Let $D$ be a smooth bounded domain in $R^{n}$. Consider the following general second order differential operator:

$$
A u=\frac{1}{2} \sum_{i, j=1}^{n} \frac{\partial}{\partial x_{i}}\left(a_{i j}(x) \frac{\partial u}{\partial x_{i}}\right)+\sum_{i=1}^{n} b_{i}(x) \frac{\partial u}{\partial x_{i}}+c(x) u(x)
$$

where $a=\left(a_{i j}(x)\right): D \rightarrow R^{n \times n}(n>2)$ is a measurable, symmetric matrixvalued function which satisfies the uniform elliptic condition

$$
\lambda_{1}|\xi|^{2} \leq \sum_{i, j=1}^{n} a_{i j}(x) \xi_{i} \xi_{j} \leq \lambda_{2}|\xi|^{2}, \forall \xi \in R^{n} \text { and } x \in D
$$

for some positive constants $\lambda_{1}, \lambda_{2}, b=\left(b_{1}, \ldots b_{n}\right),: D \rightarrow R^{n}$ and $c: D \rightarrow R$ are bounded measurable functions. Set $H=L^{2}(D)$. Let $H_{0}^{1,2}(D)$ denote the Sobolev space of order one with zero boundary condition. In view of (4.1) and the boundedness of $b$ and $c(x)$, for $u \in H_{0}^{1,2}(D)$ we have

$$
\begin{aligned}
-<A u, u>= & \frac{1}{2} \int_{D}\left(\sum_{i, j=1}^{n} a_{i j}(x) \frac{\partial u}{\partial x_{i}} \frac{\partial u}{\partial x_{j}}\right) d x \\
& -\sum_{i=1}^{n} \int_{D} b_{i}(x) \frac{\partial u}{\partial x_{i}} u(x) d x-\int_{D} c(x) u(x)^{2} d x \\
\geq & \frac{1}{2} \lambda_{1} \int_{D}|\nabla u|^{2}(x) d x-\frac{1}{4} \lambda_{1} \int_{D}|\nabla u|^{2}(x) d x-C_{\lambda_{1}} \int_{D} u^{2}(x) d x \\
= & \frac{1}{4} \lambda_{1} \int_{D}|\nabla u|^{2}(x) d x-C_{\lambda_{1}} \int_{D} u^{2}(x) d x
\end{aligned}
$$

Given a positive constant $\delta$, denote by $D([0, \delta], H)$ the space of all càdlàg paths from $[0, \delta]$ into $H$. For a path $X(\cdot): \mathbb{R}_{+} \rightarrow H, X_{t}$ will denote the function defined by $X_{t}(s)=X(t+s)$ for $s \in[0, \delta]$. Put $\mathcal{H}=L^{2}(R \rightarrow H ; \nu)$. Consider the $L^{2}$ spaces $V_{1}:=L^{2}([0, \delta] \rightarrow H, d s)$ and $V_{2}:=L^{2}([0, \delta] \rightarrow \mathcal{H}, d s)$. Let

$$
F: \mathbb{R}_{+} \times H \times H \times V_{1} \times H \times H \times V_{1} \times \mathcal{H} \times \mathcal{H} \times V_{2} \times \Omega \rightarrow H
$$

be a predictable function. Introduce the following Lipschitz condition: There exists a constant $C$ such that

$$
\begin{aligned}
& \left|F\left(t, p_{1}, p_{2}, p, q_{1}, q_{2}, q, r_{1}, r_{2}, r, \omega\right)-F\left(t, \bar{p}_{1}, \bar{p}_{2}, \bar{p}, \bar{q}_{1}, \bar{q}_{2}, \bar{q}, \bar{r}_{1}, \bar{r}_{2}, \bar{r}, \omega\right)\right|_{H} \\
& \quad \leq C\left(\left|p_{1}-\bar{p}_{1}\right|_{H}+\left|p_{2}-\bar{p}_{2}\right|_{H}+|p-\bar{p}|_{V_{1}}+\left|q_{1}-\bar{q}_{1}\right|_{H}+\left|q_{2}-\bar{q}_{2}\right|_{H}\right. \\
& \left.\quad+|q-\bar{q}|_{V_{1}}+\left|r_{1}-\bar{r}_{1}\right|_{\mathcal{H}}+\left|r_{2}-\bar{r}_{2}\right|_{\mathcal{H}}+|r-\bar{r}|_{V_{2}}\right) .
\end{aligned}
$$




\subsection{First existence and uniqueness theorem}

We first consider the following time-advanced backward stochastic partial differential equation (BSPDE) in the unknown $\mathcal{F}_{t}$ adapted processes $(p(t), q(t), r(t, z)) \in$ $H \times H \times \mathcal{H}$ :

$$
\begin{aligned}
& d p(t)=-A p(t) d t \\
& +E\left[F \left(t, p(t), p(t+\delta) \chi_{[0, T-\delta]}(t), p_{t} \chi_{[0, T-\delta]}(t), q(t), q(t+\delta) \chi_{[0, T-\delta]}(t),\right.\right. \\
& \left.\left.q_{t} \chi_{[0, T-\delta]}(t), r(t), r(t+\delta) \chi_{[0, T-\delta]}(t), r_{t} \chi_{[0, T-\delta]}(t)\right) \mid \mathcal{F}_{t}\right] d t \\
& +q(t) d B(t)+\int_{\mathbb{R}} r(t, z) \tilde{N}(d t, d z) ; t \in[0, T] \\
& \quad p(T)=G
\end{aligned}
$$

where $G$ is a given $H$-valued $\mathcal{F}_{T}$-measurable random variable.

Note that the time-advanced BSPDE (2.7)-(2.8) for the adjoint processes of the Hamiltonian is of this form.

For this type of time-advanced BSPDEs we have the following result:

Theorem 4.1 Assume that $E\left[G^{2}\right]<\infty$ and that condition (4.3) is satisfied. Then the BSPDE (4.4)-(4.5) has a unique solution $p(t), q(t), r(t, z))$ such that

$$
E\left[\int_{0}^{T}\left\{|p(t)|_{H}^{2}+|q(t)|_{H}^{2}+\int_{\mathbb{R}}|r(t, z)|_{H}^{2} \nu(d z)\right\} d t\right]<\infty
$$

Moreover, the solution can be found by inductively solving a sequence of BSPDEs backwards as follows:

Step 0: In the interval $[T-\delta, T]$ we let $p(t), q(t)$ and $r(t, z)$ be defined as the solution of the classical BSPDE (see [19])

$$
\begin{gathered}
d p(t)=-A p(t) d t+F(t, p(t), 0,0, q(t), 0,0, r(t, z), 0,0) d t \\
+q(t) d B(t)+\int_{\mathbb{R}} r(t, z) \tilde{N}(d t, d z) ; t \in[T-\delta, T] \\
p(T)=G .
\end{gathered}
$$


Step $k ; k \geq 1$ : If the values of $(p(t), q(t), r(t, z))$ have been found for $t \in[T-k \delta, T-(k-1) \delta]$, then if $t \in[T-(k+1) \delta, T-k \delta]$ the values of $p(t+\delta), p_{t}, q(t+\delta), q_{t}, r(t+\delta, z)$ and $r_{t}$ are known and hence the BSPDE

$$
\begin{aligned}
& d p(t)=-A p(t) d t \\
& +E\left[F\left(t, p(t), p(t+\delta), p_{t}, q(t), q(t+\delta), q_{t}, r(t), r(t+\delta), r_{t}\right) \mid \mathcal{F}_{t}\right] d t \\
& +q(t) d B(t)+\int_{\mathbb{R}} r(t, z) \tilde{N}(d t, d z) ; t \in[T-(k+1) \delta, T-k \delta] \\
& \quad p(T-k \delta)=\text { the value found in Step } k-1
\end{aligned}
$$

has a unique solution in $[T-(k+1) \delta, T-k \delta]$.

We proceed like this until $k$ is such that $T-(k+1) \delta \leq 0<T-k \delta$ and then we solve the corresponding BSPDE on the interval $[0, T-k \delta]$.

Proof. The proof follows directly from the above inductive procedure. The estimate (4.6) is a consequence of known estimates for classical BSPDEs.

\subsection{Second existence and uniqueness theorem}

Next, we consider the following backward stochastic partial differential equa-

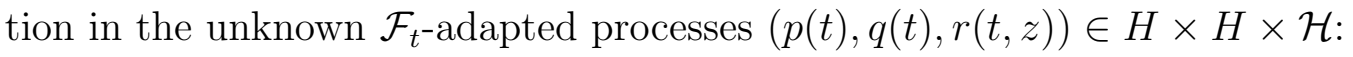

$$
\begin{gathered}
d p(t)=-A p(t) d t \\
+E\left[F\left(t, p(t), p(t+\delta), p_{t}, q(t), q(t+\delta), q_{t}, r(t), r(t+\delta), r_{t}\right) \mid \mathcal{F}_{t}\right] d t \\
+q(t) d B_{t}+\int_{\mathbb{R}} r(t, z) \tilde{N}(d t, d z), \quad t \in[0, T] \\
\quad p(t)=G(t), \quad t \in[T, T+\delta] .
\end{gathered}
$$

where $G$ is a given $H$-valued continuous $\mathcal{F}_{t^{-}}$adapted stochastic process.

Theorem 4.2 Assume $E\left[\sup _{T \leq t \leq T+\delta}|G(t)|_{H}^{2}\right]<\infty$ and that condition (4.3) is satisfied. Then the backward stochastic partial differential equation (4.11) admits a unique solution $(p(t), q(t), r(t, z))$ such that

$$
E\left[\int_{0}^{T}\left\{|p(t)|_{H}^{2}+|q(t)|_{H}^{2}+\int_{\mathbb{R}}|r(t, z)|_{H}^{2} \nu(d z)\right\} d t\right]<\infty .
$$

Proof. 
Step 1 Assume $F$ is independent of $p_{1}, p_{2}$ and $p$. Set $q^{0}(t):=0, r^{0}(t, z)=0$. For $n \geq 1$, define $\left(p^{n}(t), q^{n}(t), r^{n}(t, z)\right)$ to be the unique solution to the following backward stochastic partial differential equation:

$$
\begin{aligned}
d p^{n}(t) & =-A p^{n}(t) d t \\
& +E\left[F\left(t, q^{n-1}(t), q^{n-1}(t+\delta), q_{t}^{n-1}, r^{n-1}(t, \cdot), r^{n-1}(t+\delta, \cdot), r_{t}^{n-1}(\cdot)\right) \mid \mathcal{F}_{t}\right] d t \\
& +q^{n}(t) d B_{t}+r^{n}(t, z) \tilde{N}(d t, d z), \quad t \in[0, T] \\
& \quad p^{n}(t)=G(t) \quad t \in[T, T+\delta] .
\end{aligned}
$$

It is a consequence of the martingale representation theorem that the above equation admits a unique solution, see, e.g. [19]. We extend $q^{n}, r^{n}$ to $[0, T+\delta]$ by setting $q^{n}(s)=0, r^{n}(s, z)=0$ for $T \leq s \leq T+\delta$. We are going to show that $\left(p^{n}(t), q^{n}(t), r^{n}(t, z)\right)$ forms a Cauchy sequence. By Itô's formula, we have

$$
\begin{aligned}
0= & \left|p^{n+1}(T)-p^{n}(T)\right|_{H}^{2} \\
= & \left|p^{n+1}(t)-p^{n}(t)\right|_{H}^{2}-2 \int_{t}^{T}<A\left(p^{n+1}(s)-p^{n}(s)\right), p^{n+1}(s)-p^{n}(s)>d s \\
+ & 2 \int_{t}^{T}<p^{n+1}(s)-p^{n}(s),\left(E\left[F\left(s, q^{n}(s), q^{n}(s+\delta), q_{s}^{n}, r^{n}(s, \cdot), r^{n}(s+\delta, \cdot), r_{s}^{n}(\cdot)\right) \mid \mathcal{F}_{s}\right]\right. \\
& \left.\quad-E\left[F\left(s, q^{n-1}(s), q^{n-1}(s+\delta), q_{s}^{n-1}, r^{n-1}(s, \cdot), r^{n-1}(s+\delta, \cdot), r_{s}^{n-1}(\cdot)\right) \mid \mathcal{F}_{s}\right]\right)>_{H} d s \\
+ & \int_{t}^{T} \int_{\mathbb{R}}\left|r^{n+1}(s, z)-r^{n}(s, z)\right|_{H}^{2} d s \nu(d z)+\int_{t}^{T}\left|q^{n+1}(s)-q^{n}(s)\right|_{H}^{2} d s \\
+ & 2 \int_{t}^{T}<p^{n+1}(s)-p^{n}(s), q^{n+1}(s)-q^{n}(s)>_{H} d B_{s} \\
+ & \int_{t}^{T} \int_{\mathbb{R}}\left\{\left|r^{n+1}(s, z)-r^{n}(s, z)\right|_{H}^{2}\right. \\
& \left.+2<p^{n+1}(s-)-p^{n}(s-), r^{n+1}(s, z)-r^{n}(s, z)>_{H}\right\} \tilde{N}(d s, d z) \quad \text { (4.14) }
\end{aligned}
$$


Rearranging terms, in view of (4.3) and (4.2) we get

$$
\begin{aligned}
& E\left[\left|p^{n+1}(t)-p^{n}(t)\right|_{H}^{2}\right]+\frac{1}{2} E\left[\int_{t}^{T}\left|\nabla\left(p^{n+1}(s)-p^{n}(s)\right)\right|_{H}^{2} d s\right] \\
&+E\left[\int_{t}^{T} \int_{\mathbb{R}}\left|r^{n+1}(s, z)-r^{n}(s, z)\right|_{H}^{2} d s \nu(d z)\right]+E\left[\int_{t}^{T}\left|q^{n+1}(s)-q^{n}(s)\right|_{H}^{2} d s\right] \\
& \leq 2 E\left[\int_{t}^{T} \mid<p^{n+1}(s)-p^{n}(s), E\left[F\left(s, q^{n}(s), q^{n}(s+\delta), r^{n}(s, \cdot), r^{n}(s+\delta, \cdot)\right)\right.\right. \\
&\left.\left.+F\left(s, q^{n-1}(s), q^{n-1}(s+\delta), r^{n-1}(s, \cdot), r^{n-1}(s+\delta, \cdot)\right) \mid \mathcal{F}_{s}\right]>_{H} \mid d s\right] \\
&+C E\left[\int_{t}^{T}\left|p^{n+1}(s)-p^{n}(s)\right|_{H}^{2} d s\right] \\
& \leq C_{\varepsilon} E\left[\int_{t}^{T}\left|p^{n+1}(s)-p^{n}(s)\right|_{H}^{2} d s\right]+\varepsilon E\left[\int_{t}^{T}\left|q^{n}(s)-q^{n-1}(s)\right|_{H}^{2} d s\right] \\
&+ \varepsilon E\left[\int_{t}^{T}\left|q^{n}(s+\delta)-q^{n-1}(s+\delta)\right|_{H}^{2} d s\right]+\varepsilon E\left[\int_{t}^{T}\left(\int_{s}^{s+\delta}\left|q^{n}(u)-q^{n-1}(u)\right|_{H}^{2} d u\right) d s\right] \\
&+\varepsilon E\left[\int_{t}^{T}\left|r^{n}(s)-r^{n-1}(s)\right|_{\mathcal{H}}^{2} d s\right] \\
&+\varepsilon E\left[\int_{t}^{T}\left|r^{n}(s+\delta)-r^{n-1}(s+\delta)\right|_{\mathcal{H}}^{2} d s\right]+\varepsilon E\left[\int_{t}^{T}\left(\int_{s}^{s+\delta}\left|r^{n}(u)-r^{n-1}(u)\right|_{\mathcal{H}}^{2} d u\right) d s\right]
\end{aligned}
$$

Note that

$$
E\left[\int_{t}^{T}\left|q^{n}(s+\delta)-q^{n-1}(s+\delta)\right|_{H}^{2} d s\right] \leq E\left[\int_{t}^{T}\left|q^{n}(s)-q^{n-1}(s)\right|_{H}^{2} d s\right] .
$$

Interchanging the order of integration,

$$
\begin{aligned}
& E\left[\int_{t}^{T}\left(\int_{s}^{s+\delta}\left|q^{n}(u)-q^{n-1}(u)\right|_{H}^{2} d u\right) d s\right]=E\left[\int_{t}^{T+\delta}\left|q^{n}(u)-q^{n-1}(u)\right|_{H}^{2} d u\left(\int_{u-\delta}^{u} d s\right]\right. \\
& \quad \leq \delta E\left[\int_{t}^{T}\left|q^{n}(s)-q^{n-1}(s)\right|_{H}^{2} d s\right] .
\end{aligned}
$$


Similar inequalities hold also for $r^{n}-r^{n-1}$. It follows from (4.15) that

$$
\begin{aligned}
& E\left[\left|p^{n+1}(t)-p^{n}(t)\right|_{H}^{2}\right]+\frac{1}{2} E\left[\int_{t}^{T}\left|\nabla\left(p^{n+1}(s)-p^{n}(s)\right)\right|_{H}^{2} d s\right] \\
& \quad+E\left[\int_{t}^{T} \int_{\mathbb{R}}\left|r^{n+1}(s, z)-r^{n}(s, z)\right|_{H}^{2} d s \nu(d z)\right]+E\left[\int_{t}^{T}\left|q^{n+1}(s)-q^{n}(s)\right|_{H}^{2} d s\right] \\
& \leq C_{\varepsilon} E\left[\int_{t}^{T}\left|p^{n+1}(s)-p^{n}(s)\right|_{H}^{2} d s\right]+(2+M) \varepsilon E\left[\int_{t}^{T}\left|q^{n}(s)-q^{n-1}(s)\right|_{H}^{2} d s\right] \\
& \quad+3 \varepsilon E\left[\int_{t}^{T}\left|r^{n}(s)-r^{n-1}(s)\right|_{\mathcal{H}}^{2} d s\right] .
\end{aligned}
$$

Choose $\varepsilon>0$ sufficiently small so that

$$
\begin{aligned}
& E\left[\left|p^{n+1}(t)-p^{n}(t)\right|_{H}^{2}\right]+\frac{1}{2} E\left[\int_{t}^{T}\left|\nabla\left(p^{n+1}(s)-p^{n}(s)\right)\right|_{H}^{2} d s\right] \\
& \quad+E\left[\int_{t}^{T} \int_{\mathbb{R}}\left|r^{n+1}(s, z)-r^{n}(s, z)\right|_{H}^{2} d s \nu(d z)\right]+E\left[\int_{t}^{T}\left|q^{n+1}(s)-q^{n}(s)\right|_{H}^{2} d s\right] \\
& \leq C_{\varepsilon} E\left[\int_{t}^{T}\left|p^{n+1}(s)-p^{n}(s)\right|_{H}^{2} d s\right]+\frac{1}{2} E\left[\int_{t}^{T}\left|q^{n}(s)-q^{n-1}(s)\right|_{H}^{2} d s\right] \\
& +\frac{1}{2} E\left[\int_{t}^{T}\left|r^{n}(s)-r^{n-1}(s)\right|_{\mathcal{H}}^{2} d s\right] .
\end{aligned}
$$

This implies that

$$
\begin{aligned}
-\frac{d}{d t}( & \left.e^{C_{\varepsilon} t} E\left[\int_{t}^{T}\left|p^{n+1}(s)-p^{n}(s)\right|_{H}^{2} d s\right]\right)+\frac{1}{2} e^{C_{\varepsilon} t} E\left[\int_{t}^{T}\left|\nabla\left(p^{n+1}(s)-p^{n}(s)\right)\right|_{H}^{2} d s\right] \\
& +e^{C_{\varepsilon} t} E\left[\int_{t}^{T} \int_{\mathbb{R}}\left|r^{n+1}(s, z)-r^{n}(s, z)\right|_{H}^{2} d s \nu(d z)\right]+e^{C_{\varepsilon} t} E\left[\int_{t}^{T}\left|q^{n+1}(s)-q^{n}(s)\right|_{H}^{2} d s\right] \\
\leq & \frac{1}{2} e^{C_{\varepsilon} t} E\left[\int_{t}^{T}\left|q^{n}(s)-q^{n-1}(s)\right|_{H}^{2} d s\right]+\frac{1}{2} e^{C_{\varepsilon} t} E\left[\int_{t}^{T}\left|r^{n}(s)-r^{n-1}(s)\right|_{\mathcal{H}}^{2} d s\right] .
\end{aligned}
$$


Integrating the last inequality we get

$$
\begin{aligned}
& E\left[\int_{0}^{T}\left|p^{n+1}(s)-p^{n}(s)\right|_{H}^{2} d s\right]+\int_{0}^{T} d t e^{C_{\varepsilon} t} E\left[\int_{t}^{T}\left|q^{n+1}(s)-q^{n}(s)\right|_{H}^{2} d s\right] \\
& +\int_{0}^{T} d t e^{C_{\varepsilon} t} E\left[\int_{t}^{T} \int_{\mathbb{R}}\left|r^{n+1}(s, z)-r^{n}(s, z)\right|_{H}^{2} d s \nu(d z)\right] \\
& +\frac{1}{2} \int_{0}^{T} d t e^{C_{\varepsilon} t} E\left[\int_{t}^{T}\left|\nabla\left(p^{n+1}(s)-p^{n}(s)\right)\right|_{H}^{2} d s\right] \\
& \leq \frac{1}{2} \int_{0}^{T} d t e^{C_{\varepsilon} t} E\left[\int_{t}^{T}\left|q^{n}(s)-q^{n-1}(s)\right|_{H}^{2} d s\right]+\frac{1}{2} \int_{0}^{T} d t e^{C_{\varepsilon} t} E\left[\int_{t}^{T}\left|r^{n}(s)-r^{n-1}(s)\right|_{\mathcal{H}}^{2} d s\right]
\end{aligned}
$$

In particular,

$$
\begin{aligned}
& \int_{0}^{T} d t e^{C_{\varepsilon} t} E\left[\int_{t}^{T} \int_{\mathbb{R}}\left|r^{n+1}(s, z)-r^{n}(s, z)\right|_{H}^{2} d s \nu(d z)\right] \\
& \quad+\int_{0}^{T} d t e^{C_{\varepsilon} t} E\left[\int_{t}^{T}\left|q^{n+1}(s)-q^{n}(s)\right|_{H}^{2} d s\right] \\
& \quad \leq \frac{1}{2} \int_{0}^{T} d t e^{C_{\varepsilon} t} E\left[\int_{t}^{T}\left|q^{n}(s)-q^{n-1}(s)\right|_{H}^{2} d s\right]+\frac{1}{2} \int_{0}^{T} d t e^{C_{\varepsilon} t} E\left[\int_{t}^{T}\left|r^{n}(s)-r^{n-1}(s)\right|_{\mathcal{H}}^{2} d s\right]
\end{aligned}
$$

This yields

$$
\begin{aligned}
& \int_{0}^{T} d t e^{C_{\varepsilon} t} E\left[\int_{t}^{T} \int_{\mathbb{R}}\left|r^{n+1}(s, z)-r^{n}(s, z)\right|_{H}^{2} d s \nu(d z)\right]+\int_{0}^{T} d t e^{C_{\varepsilon} t} E\left[\int_{t}^{T}\left|q^{n+1}(s)-q^{n}(s)\right|_{H}^{2} d s\right] \\
& \quad \leq\left(\frac{1}{2}\right)^{n} C
\end{aligned}
$$

for some constant $C$. It follows from (4.21) that

$$
E\left[\int_{0}^{T}\left|p^{n+1}(s)-p^{n}(s)\right|_{H}^{2} d s\right] \leq\left(\frac{1}{2}\right)^{n} C .
$$

(4.18) and ((4.21) further gives

$$
\begin{aligned}
& E\left[\int_{0}^{T} \int_{\mathbb{R}}\left|r^{n+1}(s, z)-r^{n}(s, z)\right|_{H}^{2} d s \nu(d z)\right]+E\left[\int_{0}^{T}\left|q^{n+1}(s)-q^{n}(s)\right|_{H}^{2} d s\right] \\
\leq & \left(\frac{1}{2}\right)^{n} C n C_{\varepsilon} .
\end{aligned}
$$


In view of (4.18), (4.21) and (4.22), we conclude that there exist progressively measurable processes $(p(t), q(t), r(t, z))$ such that

$$
\begin{aligned}
& \lim _{n \rightarrow \infty} E\left[\left|p^{n}(t)-p(t)\right|_{H}^{2}\right]=0, \\
& \lim _{n \rightarrow \infty} \int_{0}^{T} E\left[\left|p^{n}(t)-p(t)\right|_{H}^{2}\right] d t=0, \\
& \lim _{n \rightarrow \infty} \int_{0}^{T} E\left[\left|\nabla\left(p^{n}(t)-p(t)\right)\right|_{H}^{2}\right] d t=0, \\
& \lim _{n \rightarrow \infty} \int_{0}^{T} E\left[\left|q^{n}(t)-q(t)\right|_{H}^{2}\right] d t=0, \\
& \lim _{n \rightarrow \infty} \int_{0}^{T} \int_{\mathbb{R}} E\left[\left|r^{n}(t, z)-r(t, z)\right|_{H}^{2}\right] \nu(d z) d t=0 .
\end{aligned}
$$

Letting $n \rightarrow \infty$ in (4.13) we see that $(p(t), q(t), r(t, z))$ satisfies

$$
\begin{aligned}
p(t) & -\int_{t}^{T} A p(s) d s \\
& +\int_{t}^{T} E\left[F\left(s, q(s), q(s+\delta), q_{s}, r(s, \cdot), r(s+\delta, \cdot), r_{s}(\cdot)\right) \mid \mathcal{F}_{s}\right] d s \\
& +\int_{t}^{T} q(s) d B_{s}+\int_{t}^{T} \int_{\mathbb{R}} r(s, z) \tilde{N}(d s, d z)=g(T)
\end{aligned}
$$

i.e., $(p(t), q(t), r(t, z))$ is a solution. Uniqueness follows easily from Ito's formula, a similar calculation of deducing (4.14) and (4.15), and Gronwall's Lemma.

Step 2. General case. Let $p^{0}(t)=0$. For $n \geq 1$, define $\left(p^{n}(t), q^{n}(t), r^{n}(t, z)\right)$ to be the unique solution of the following BSDE:

$$
\begin{gathered}
d p^{n}(t)=-A p^{n}(t) d t+q^{n}(t) d B_{t}+r^{n}(t, z) \tilde{N}(d t, d z) \\
+E\left[F\left(t, p^{n-1}(t), p^{n-1}(t+\delta), p_{t}^{n-1}, q^{n}(t), q^{n}(t+\delta), q_{t}^{n}, r^{n}(t, \cdot), r^{n}(t+\delta, \cdot), r_{t}^{n}(\cdot)\right) \mid \mathcal{F}_{t}\right] d t \\
p^{n}(t)=G(t) ; \quad t \in[T, T+\delta] .
\end{gathered}
$$

The existence of $\left(p^{n}(t), q^{n}(t), r^{n}(t, z)\right)$ is proved in Step 1. By the same 
arguments leading to (4.18), we deduce that

$$
\begin{aligned}
& E\left[\left|p^{n+1}(t)-p^{n}(t)\right|_{H}^{2}\right]+\frac{1}{2} E\left[\int_{t}^{T} \int_{\mathbb{R}}\left|r^{n+1}(s, z)-r^{n}(s, z)\right|_{H}^{2} d s \nu(d z)\right] \\
& +\frac{1}{2} E\left[\int_{t}^{T}\left|q^{n+1}(s)-q^{n}(s)\right|_{H}^{2} d s\right]+\frac{1}{2} \lambda_{1} E\left[\int_{t}^{T}\left|\nabla\left(p^{n+1}(s)-p^{n}(s)\right)\right|_{H}^{2} d s\right] \\
& \quad \leq C E\left[\int_{t}^{T}\left|p^{n+1}(s)-p^{n}(s)\right|_{H}^{2} d s\right]+\frac{1}{2} E\left[\int_{t}^{T}\left|p^{n}(s)-p^{n-1}(s)\right|_{H}^{2} d s\right]
\end{aligned}
$$

This implies that

$-\frac{d}{d t}\left(e^{C t} E\left[\int_{t}^{T}\left|p^{n+1}(s)-p^{n}(s)\right|_{H}^{2} d s\right]\right) \leq \frac{1}{2} e^{C t} E\left[\int_{t}^{T}\left|p^{n}(s)-p^{n-1}(s)\right|_{H}^{2} d s\right]$

Integrating (4.29) from $u$ to $T$ we get

$$
\begin{aligned}
& E\left[\int_{u}^{T}\left|p^{n+1}(s)-p^{n}(s)\right|_{H}^{2} d s\right] \leq \frac{1}{2} \int_{u}^{T} d t e^{C(t-u)} E\left[\int_{t}^{T}\left|p^{n}(s)-p^{n-1}(s)\right|_{H}^{2} d s\right] \\
& \leq e^{C T} \int_{u}^{T} d t E\left[\int_{t}^{T}\left|p^{n}(s)-p^{n-1}(s)\right|_{H}^{2} d s\right] .
\end{aligned}
$$

Iterating the above inequality we obtain that

$$
E\left[\int_{0}^{T}\left|p^{n+1}(s)-p^{n}(s)\right|_{H}^{2} d s\right] \leq \frac{e^{C n T} T^{n}}{n !}
$$

Using above inequality and a similar argument as in Step 1, it can be shown that $\left(p^{n}(t), q^{n}(t), r^{n}(t, z)\right)$ converges to some limit $(p(t), q(t), r(t, z))$, which is the unique solution of equation (4.11).

Theorem 4.3 Assume $E\left[\sup _{T \leq t \leq T+\delta}|G(t)|_{H}^{2 \alpha}\right]<\infty$ for some $\alpha>1$ and that the following condition hold:

$$
\begin{aligned}
& \left|F\left(t, p_{1}, p_{2}, p, q_{1}, q_{2}, q, r_{1}, r_{2}, r\right)-F\left(t, \bar{p}_{1}, \bar{p}_{2}, \bar{p}, \bar{q}_{1}, \bar{q}_{2}, \bar{q}, \bar{r}_{1}, \bar{r}_{2}, \bar{r}\right)\right| \\
& \quad \leq \quad C\left(\left|p_{1}-\bar{p}_{1}\right|_{H}+\left|p_{2}-\bar{p}_{2}\right|_{H}+\sup _{0 \leq s \leq \delta}|p(s)-\bar{p}(s)|_{H}+\left|q_{1}-\bar{q}_{1}\right|_{H}+\left|q_{2}-\bar{q}_{2}\right|_{H}+|q-\bar{q}|_{V_{1}}\right. \\
& \left.\quad+\left|r_{1}-\bar{r}_{1}\right|_{\mathcal{H}}+\left|r_{2}-\bar{r}_{2}\right|_{\mathcal{H}}+|r-\bar{r}|_{V_{2}}\right) .
\end{aligned}
$$


Then the BSPDE (4.11) admits a unique solution $(p(t), q(t), r(t, z))$ such that

$$
E\left[\sup _{0 \leq t \leq T}|p(t)|_{H}^{2 \alpha}+\int_{0}^{T}\left\{|q|_{H}^{2}(t)+\int_{\mathbb{R}}|r|_{H}^{2}(t, z) \nu(d z)\right\} d t\right]<\infty .
$$

Proof.

Step 1. Assume $F$ is independent of $p_{1}, p_{2}$ and $p$. In this case condition (4.31) reduces to assumption (4.3). By the Step 1 in the proof of Theorem 4.2 , there is a unique solution $(p(t), q(t), r(t, z))$ to equation (4.11).

Step 2. General case. Let $p^{0}(t)=0$. For $n \geq 1$, define $\left(p^{n}(t), q^{n}(t), r^{n}(t, z)\right)$ to be the unique solution of the following BSDE:

$$
\begin{aligned}
& d p^{n}(t)=-A p^{n}(t) d t+q^{n}(t) d B_{t}+r^{n}(t, z) \tilde{N}(d t, d z) \\
& +E\left[F\left(t, p^{n-1}(t), p^{n-1}(t+\delta), p_{t}^{n-1}, q^{n}(t), q^{n}(t+\delta), q_{t}^{n}, r^{n}(t, \cdot), r^{n}(t+\delta, \cdot), r_{t}^{n}(\cdot)\right) \mid \mathcal{F}_{t}\right] d t
\end{aligned}
$$

$$
p^{n}(t)=G(t), \quad t \in[T, T+\delta] .
$$

By Step 1, $\left(p^{n}(t), q^{n}(t), r^{n}(t, z)\right)$ exists. We are going to show that $\left(p^{n}(t), q^{n}(t), r^{n}(t, z)\right)$ forms a Cauchy sequence. Using Itô's formula, we have

$$
\begin{aligned}
& \left|p^{n+1}(t)-p^{n}(t)\right|_{H}^{2}+\int_{t}^{T} \int_{\mathbb{R}}\left|r^{n+1}(s, z)-r^{n}(s, z)\right|_{H}^{2} d s \nu(d z)+\int_{t}^{T}\left|q^{n+1}(s)-q^{n}(s)\right|_{H}^{2} d s \\
& -2 \int_{t}^{T}<A p^{n}(s), p^{n}(s)>d s \\
& \quad=-2 \int_{t}^{T}<p^{n+1}(s)-p^{n}(s) \\
& \quad E\left[F\left(s, p^{n}(s), p^{n}(s+\delta), p_{s}^{n}, q^{n+1}(s), q^{n+1}(s+\delta), q_{s}^{n+1}, r^{n+1}(s, \cdot), r^{n+1}(s+\delta, \cdot), r_{s}^{n+1}(\cdot)\right)\right. \\
& \left.-F\left(s, p^{n-1}(s), p^{n-1}(s+\delta), p_{s}^{n-1}, q^{n}(s), q^{n}(s+\delta), q_{s}^{n}, r^{n}(s, \cdot), r^{n}(s+\delta, \cdot), r_{s}^{n}(\cdot)\right) \mid \mathcal{F}_{s}\right]>_{H} d s \\
& -2 \int_{t}^{T}<p^{n+1}(s)-p^{n}(s), q^{n+1}(s)-q^{n}(s)>d B_{s} \\
& -\int_{t}^{T} \int_{\mathbb{R}}\left[\left|r^{n+1}(s, z)-r^{n}(s, z)\right|_{H}^{2}+2<p^{n+1}(s-)-p^{n}(s-), r^{n+1}(s, z)-r^{n}(s, z)>_{H}\right] \tilde{N}(d s, d z)
\end{aligned}
$$


Take conditional expectation with respect to $\mathcal{F}_{t}$, take the supremum over the interval $[u, T]$ and use the condition (4.31) to get

$$
\begin{aligned}
\sup _{u \leq t \leq T}\left|p^{n+1}(t)-p^{n}(t)\right|_{H}^{2}+\sup _{u \leq t \leq T} E\left[\int_{t}^{T}\left|q^{n+1}(s)-q^{n}(s)\right|_{H}^{2} d s \mid \mathcal{F}_{t}\right] \\
+\sup _{u \leq t \leq T} E\left[\int_{t}^{T} \int_{\mathbb{R}}\left|r^{n+1}(s, z)-r^{n}(s, z)\right|_{H}^{2} d s \nu(d z) \mid \mathcal{F}_{t}\right] \\
+\sup _{u \leq t \leq T} E\left[\int_{t}^{T}\left|\nabla\left(p^{n+1}(s)-p^{n}(s)\right)\right|_{H}^{2} d s \nu(d z) \mid \mathcal{F}_{t}\right] \\
\leq C_{\varepsilon} \sup _{u \leq t \leq T} E\left[\int_{u}^{T}\left|p^{n+1}(s)-p^{n}(s)\right|_{H}^{2} d s \mid \mathcal{F}_{t}\right] \\
+C_{1} \varepsilon \sup _{u \leq t \leq T} E\left[\int_{u}^{T}\left|p^{n}(s)-p^{n-1}(s)\right|_{H}^{2} d s \mid \mathcal{F}_{t}\right] \\
\quad+C_{2} \varepsilon \sup _{u \leq t \leq T} E\left[\int_{u}^{T} E\left[\sup _{s \leq v \leq T}\left|p^{n}(v)-p^{n-1}(v)\right|_{H}^{2} \mid \mathcal{F}_{s}\right] d s \mid \mathcal{F}_{t}\right] \\
+C_{3} \varepsilon \sup _{u \leq t \leq T} E\left[\int_{t}^{T}\left|q^{n+1}(s)-q^{n}(s)\right|_{H}^{2} d s \mid \mathcal{F}_{t}\right] \\
+C_{4} \varepsilon \sup _{u \leq t \leq T} E\left[\int_{t}^{T} \int_{\mathbb{R}}\left|r^{n+1}(s, z)-r^{n}(s, z)\right|_{H}^{2} d s \nu(d z) \mid \mathcal{F}_{t}\right]
\end{aligned}
$$

Choosing $\varepsilon>0$ such that $C_{3} \varepsilon<1$ and $C_{4} \varepsilon<1$ it follows from (4.34) that

$$
\begin{aligned}
\sup _{u \leq t \leq T}\left|p^{n+1}(t)-p^{n}(t)\right|_{H}^{2} \leq C_{\varepsilon} \sup _{u \leq t \leq T} E\left[\int_{u}^{T}\left|p^{n+1}(s)-p^{n}(s)\right|_{H}^{2} d s \mid \mathcal{F}_{t}\right] \\
+\left(C_{1}+C_{2}\right) \varepsilon \sup _{u \leq t \leq T} E\left[\int_{u}^{T} E\left[\sup _{s \leq v \leq T}\left|p^{n}(v)-p^{n-1}(v)\right|_{H}^{2} \mid \mathcal{F}_{s}\right] d s \mid \mathcal{F}_{t}\right]
\end{aligned}
$$

Note that $E\left[\int_{u}^{T}\left|p^{n+1}(s)-p^{n}(s)\right|_{H}^{2} d s \mid \mathcal{F}_{t}\right]$ and $E\left[\int_{u}^{T} E\left[\sup _{s \leq v \leq T}\left|p^{n}(v)-p^{n-1}(v)\right|_{H}^{2} \mid \mathcal{F}_{s}\right] d s \mid \mathcal{F}_{t}\right]$ are right-continuous martingales on $[0, T]$ with terminal random variables $\int_{u}^{T}\left|p^{n+1}(s)-p^{n}(s)\right|_{H}^{2} d s$ and $\int_{u}^{T} E\left[\sup _{s \leq v \leq T}\left|p^{n}(v)-p^{n-1}(v)\right|_{H}^{2} \mid \mathcal{F}_{s}\right] d s$. Thus for 
$\alpha>1$, we have

$$
\begin{aligned}
& E\left[\left(\sup _{u \leq t \leq T} E\left[\int_{u}^{T}\left|p^{n+1}(s)-p^{n}(s)\right|_{H}^{2} d s \mid \mathcal{F}_{t}\right]\right)^{\alpha}\right] \leq c_{\alpha} E\left[\left(\int_{u}^{T}\left|p^{n+1}(s)-p^{n}(s)\right|_{H}^{2} d s\right)^{\alpha}\right] \\
& \quad \leq c_{T, \alpha} E\left[\int_{u}^{T} \sup _{s \leq v \leq T}\left|p^{n+1}(v)-p^{n}(v)\right|_{H}^{2 \alpha} d s\right]
\end{aligned}
$$

and

$$
\begin{aligned}
E & {\left[\left(\sup _{u \leq t \leq T} E\left[\int_{u}^{T} E\left[\sup _{s \leq v \leq T}\left|p^{n}(v)-p^{n-1}(v)\right|_{H}^{2} \mid \mathcal{F}_{s}\right] d s \mid \mathcal{F}_{t}\right]\right)^{\alpha}\right] } \\
& \leq c_{T, \alpha} E\left[\int_{u}^{T} E\left[\sup _{s \leq v \leq T}\left|p^{n}(v)-p^{n-1}(v)\right|_{H}^{2 \alpha} \mid \mathcal{F}_{s}\right] d s\right] \\
& \leq c_{T, \alpha} E\left[\int_{u}^{T} \sup _{s \leq v \leq T}\left|p^{n}(v)-p^{n-1}(v)\right|_{H}^{2 \alpha} d s\right] .
\end{aligned}
$$

(4.35), (4.36) and (4.37) yield that for $\alpha>1$,

$$
\begin{aligned}
& E\left[\sup _{u \leq t \leq T}\left|p^{n+1}(t)-p^{n}(t)\right|_{H}^{2 \alpha}\right] \leq C_{1, \alpha} E\left[\int_{u}^{T} \sup _{s \leq v \leq T}\left|p^{n+1}(v)-p^{n}(v)\right|_{H}^{2 \alpha} d s\right] \\
& \quad+C_{2, \alpha} E\left[\int_{u}^{T} \sup _{s \leq v \leq T}\left|p^{n}(v)-p^{n-1}(v)\right|_{H}^{2 \alpha} d s\right] .
\end{aligned}
$$

Put

$$
g_{n}(u)=E\left[\int_{u}^{T} \sup _{t \leq s \leq T}\left|p^{n}(s)-p^{n-1}(s)\right|_{H}^{2 \alpha}\right] .
$$

(4.38) implies that

$$
-\frac{d}{d t}\left(e^{C_{1, \alpha} u} g_{n+1}(u)\right) \leq e^{C_{1, \alpha} u} C_{2, \alpha} g_{n}(u) .
$$

Integrating (4.39) from $t$ to $T$ we get

$$
g_{n+1}(t) \leq c_{2, \alpha} \int_{t}^{T} e^{C_{1, \alpha}(s-t)} g_{n}(s) d s \leq C_{2, \alpha} e^{C_{1, \alpha} T} \int_{t}^{T} g_{n}(s) d s .
$$

Iterating the above inequality we obtain that

$$
E\left[\int_{0}^{T} \sup _{t \leq s \leq T}\left|p^{n+1}(s)-p^{n}(s)\right|_{H}^{2 \alpha} d t\right] \leq \frac{e^{C n T} T^{n}}{n !} .
$$


Using above inequality and a similar argument as in step 1, we can show that $\left(p^{n}(t), q^{n}(t), r^{n}(t, z)\right)$ converges to some limit $(p(t), q(t), r(t, z))$, which is the unique solution of equation (4.11).

\section{Application}

To illustrate our results, we now apply them to the optimal harvesting problem presented in the Introduction. In this case the state is given by the reaction-diffusion equation (1.1) and the performance functional is given by (1.2). Therefore, by (2.7) the corresponding Hamiltonian is

$$
\begin{aligned}
H(t, x, y, z, a, c, p, q, r(\cdot)) & =U_{1}(t, x, c)+(\alpha y+\beta z-c) p \\
& +\sigma_{0} y q+\int_{\mathbb{R}} \gamma_{0}(\zeta) r(\zeta) y \nu(d \zeta)
\end{aligned}
$$

The BSPDE for the adjoint processes $p(t, x), q(t, x), r(t, x, \zeta)$ is, by $(2.9)$,

$$
\begin{aligned}
d p(t, x)= & -\left\{\frac{1}{2} \Delta p(t, x)+\alpha p(t, x)+\sigma_{0} q(t, x)+\int_{\mathbb{R}} \gamma_{0}(\zeta) r(t, x, \zeta) \nu(d \zeta)\right. \\
& \left.+\beta E\left[p(t+\delta, x) \mid \mathcal{F}_{t}\right] \chi_{[0, T-\delta]}(t)\right\} d t \\
& +q(t, x) d B(t)+\int_{\mathbb{R}} r(t, x, \zeta) \tilde{N}(d t, d \zeta) ; 0 \leq t \leq T \\
p(T, x)= & U_{2}^{\prime}(x, Y(T, x)) ; x \in D \quad\left(\text { where } U_{2}^{\prime}(x, y)=\frac{\partial}{\partial y} U_{2}(x, y)\right) \\
p(t, x)= & 0 ; \quad(t, x) \in(0, T) \times \partial D .
\end{aligned}
$$

Maximizing the conditional Hamiltonian with respect to $c$ gives the first order condition:

$$
\frac{\partial U_{1}}{\partial c}(t, x, c(t, x))=E\left[p(t, x) \mid \mathcal{E}_{t}\right] ; \quad(t, x) \in[0, T] \times D
$$

So, assuming that $c \rightarrow \frac{\partial U_{1}}{\partial c}(t, x, c)$ is strictly decreasing, to find the optimal $c=c^{*}(t, x)$ it suffices to find the solution $p(t, x)$ of $(5.2)$. To this end, we proceed inductively, as in Theorem 4.1: 
Step 0: In the interval $[T-\delta, T]$ we solve the following BSPDE for $p(t, x)$, $q(t, x), r(t, x, \zeta)$ :

$$
\begin{aligned}
d p(t, x)= & -\left\{\frac{1}{2} \Delta p(t, x)+\alpha p(t, x)+\sigma_{0} q(t, x)+\int_{\mathbb{R}} \gamma_{0}(\zeta) r(t, x, \zeta) \nu(d \zeta)\right\} d t \\
& +q(t, x) d B(t)+\int_{\mathbb{R}} r(t, x, \zeta) \tilde{N}(d t, d \zeta) ; t \in[T-\delta, T] \\
p(T, x)= & U_{2}^{\prime}(x, Y(T, x)) ; x \in D, \quad p(t, x)=0 ; \quad(t, x) \in(0, T) \times \partial D .
\end{aligned}
$$

It is well known that this equation has a unique solution. See [19]. In fact, in this particular case the solution can be found explicitly by adopting the procedure in Example 1.1 of [19]. as follows:

Choose $\mathcal{F}_{t}$-predictable processes $\Theta_{0}(t, x)$ and $\Theta_{1}(t, x, \zeta)$ such that

$$
\begin{aligned}
& q(t, x) \Theta_{0}(t, x)+\int_{\mathbb{R}} r(t, x, \zeta) \Theta_{1}(t, x, \zeta) \nu(d \zeta) \\
= & q(t, x) \sigma_{0}+\int_{\mathbb{R}} r(t, x, \zeta) \gamma_{0}(\zeta) \nu(d \zeta)
\end{aligned}
$$

An obvious choice is

$$
\Theta_{0}(t, x)=\sigma_{0} \quad \Theta_{1}(t, x, \zeta)=\gamma_{0}(\zeta)
$$

Next, define the probability measure $Q$ on $\mathcal{F}_{T}$ by

$$
d Q(\omega)=Z_{T}(\omega) d P(\omega),
$$

where

$$
\left\{\begin{array}{l}
d Z_{t}=Z_{t-}(\omega)\left[-\sigma_{0} d B(t)-\int_{\mathbb{R}} \gamma_{0}(\zeta) \tilde{N}(d t, d \zeta)\right] \\
Z_{0}=1
\end{array}\right.
$$

i.e.

$$
\begin{aligned}
Z_{t}= & \exp \left(-\sigma_{0} B(t)-\frac{1}{2} \sigma_{0}^{2} t+\int_{0}^{t} \int_{\mathbb{R}} \ln \left(1-\gamma_{0}(\zeta)\right) \tilde{N}(d s, d \zeta)\right. \\
& \left.+t \int_{\mathbb{R}}\left\{\ln \left(1-\gamma_{0}(\zeta)\right)+\gamma_{0}(\zeta)\right\} \nu(d \zeta)\right) ; \quad 0 \leq t \leq T
\end{aligned}
$$

Then

$$
B_{Q}(t):=\sigma_{0} t+B(t)
$$


and

$$
\tilde{N}_{Q}(d t, d \zeta):=\gamma_{0}(\zeta) \nu(d \zeta) d t+\tilde{N}(d t, d \zeta)
$$

is a $Q$-Brownian motion and a $Q$-compensated Poisson random measure, respectively, and in terms of $B_{Q}$ and $\tilde{N}_{Q}$ the BSPDE (5.4) gets the form

$$
\begin{aligned}
d p(t, x)= & -\left\{\frac{1}{2} \Delta p(t, x)+\alpha p(t, x)\right\} d t+q(t, x) d B_{Q}(t) \\
& +\int_{\mathbb{R}} r(t, x, \zeta) \tilde{N}(d t, d \zeta) ;(t, x) \in[T-\delta, T] \times D \\
p(T, x)= & U_{2}^{\prime}(x, Y(T, x)) ; x \in D, \\
p(t, x)= & 0 ; \quad(t, x) \in(0, T) \times \partial D .
\end{aligned}
$$

See [16], chapter 1 for details.

Let

$$
R_{t} f(x)=\left\{\begin{array}{l}
\int_{\mathbb{R}}(2 \pi t)^{-\frac{1}{2}} f(y) \exp \left(-\frac{|x-y|^{2}}{2 t}\right) d y ; \quad t>0 \\
f(x) ; t=0
\end{array}\right.
$$

be the transition operator for Brownian motion, defined for all $f: \mathbb{R} \rightarrow \mathbb{R}$ such that the integral exists. Then

$$
\frac{\partial}{\partial t}\left(R_{t} f(x)\right)=\frac{1}{2} \Delta\left(R_{t} f(x)\right)
$$

Put

$$
F(x)=U_{2}^{\prime}(x, Y(T, x)) .
$$

By the Itô representation theorem there exists $\mathcal{F}_{t}$-predictable processes $g(s, x)=g(s, x, \omega)$ and $k(s, x, \zeta)=k(s, x, \zeta, \omega)$ such that

$$
E_{Q}\left[\int_{D}\left(\int_{0}^{T}\left\{g^{2}(s, x)+\int_{\mathbb{R}} k^{2}(s, x, \zeta) \nu(d \zeta)\right\} d s\right) d x\right]<\infty
$$

and

$$
F(x)=E_{Q}[F(x)]+\int_{0}^{T} g(s, x) d B_{Q}(s)+\int_{0}^{T} \int_{\mathbb{R}} k(s, x, \zeta) \tilde{N}_{Q}(d s, d \zeta) .
$$

In fact, by the $L^{2}$-extension of the Clark-Ocone formula ([1]) we have

$$
g(s, x)=E_{Q}\left[D_{s} F(x) \mid \mathcal{F}_{s}\right], \quad k(s, x, \zeta)=E_{Q}\left[D_{s, \zeta} F(x) \mid \mathcal{F}_{s}\right]
$$


where $D_{s}$ and $D_{s, \zeta}$ denotes the Malliavin derivatives with respect to $B$ and $\tilde{N}$, respectively. Define

$$
\begin{gathered}
p(t, x)=e^{\alpha(T-t)} R_{T-t}\left(\int_{0}^{t} g(s, \cdot) d B_{Q}(s)+\int_{0}^{t} \int_{\mathbb{R}} k(s, \cdot, \zeta) \tilde{N}_{Q}(d s, d \zeta)+E_{Q}[F(\cdot)]\right)(x) \\
\left.q(t, x)=e^{\alpha(T-t)} R_{T-t} g(t, \cdot)\right)(x) \\
\left.r(t, x, \zeta)=e^{\alpha(T-t)} R_{T-t}(k(s, \cdot, \zeta))(x) ;(t, x) \in[0, T] \times D .12\right)
\end{gathered}
$$

Then $(p, q, r)$ solves (5.4). This completes Step 0.

We now proceed to the next step:

Step 1 . If $[T-2 \delta, T-\delta]$ and $T-2 \delta>0$, we get by (5.2) the BSPDE

$$
\begin{aligned}
d p(t, x)= & -\left\{\frac{1}{2} \Delta p(t, x)+\alpha p(t, x)+\sigma_{0} q(t, x)\right. \\
& \left.+\int_{\mathbb{R}} \gamma_{0}(\zeta) r(t, x, \zeta) \nu(d \zeta)+\beta E\left[p(t+\delta, x) \mid \mathcal{F}_{t}\right]\right\} d t \\
& +q(t, x) d B(t)+\int_{\mathbb{R}} r(t, x, \zeta) \tilde{N}(d t, d \zeta) ;(t, x) \in[T-2 \delta, T-\delta] \times \partial D .
\end{aligned}
$$

Note that if $t \in[T-2 \delta, T-\delta]$ then $P(t+\delta, x)$, and in particular $p(T-\delta, x)$, are known by Step 0. So again we can solve the BSPDE by the method in [19].

We continue like this by induction up to and including step $j$, where $j$ is such that $T-j \delta \leq 0<T-(j-1) \delta$. This procedure leads to a unique solution $p(t, x)$ of $(5.2)$. Note, however, that this solution $p(t, x)=p^{(c)}(t, x)$ depends in a complicated way of the optimal terminal value $Y(T, x)$, which again depends on the optimal control $c(t, x)$. Therefore equation (5.3), given by

$$
\frac{\partial U_{1}}{\partial c}(t, x, c(t, x))=E\left[p(c)(t, x) \mid \mathcal{E}_{t}\right]
$$

is in general difficult to solve for the optimal control $c(t, x)$.

However, there is an important solvable special case, namely when

$$
U_{2}(x, y)=U_{2}(x, y, \omega)=k(x, \omega) y
$$

for some bounded $\mathcal{F}_{T}$-measurable random variable $k(x)=k(x, \omega) \geq 0$. In this case we get

$$
F(x, \omega)=U_{2}^{\prime}(x, Y(T, x))=k(x, \omega),
$$


which does not depend on $c$, and hence (5.15) can be solved for the optimal $c(t, x)$.

We summarize the above as follows:

Theorem 5.1 The optimal consumption rate $c(t, x)$ of the problem (1.1), (1.2), when $U_{2}(x, y)$ is of the form (5.16), is given by equation (5.15), where $p(t, x)$ is the solution of (5.2), as outlined in (5.4)-(5.14).

It is interesting to note how the delay affects the optimal harvesting policy: Using inductively at each step above the comparison theorem for BSPDEs [18] we obtain the following:

Corollary 5.2 For $0<\delta_{1}<\delta_{2}$ let $p_{\delta_{i}}(t, x)$ be the solution of the BSPDE (5.2) corresponding to $\delta_{i} ; i=1,2$, with

$$
p_{\delta_{i}}(T, x)=U_{2}^{\prime}(x, Y(T, x))=k(x, \omega)
$$

as in Theorem 5.1. Assume that $\beta \geq 0$. Then

(i) $p_{\delta_{2}}(t, x) \leq p_{\delta_{1}}(t, x) ;(t, x) \in[0, T] \times D$.

Hence, by (5.15), the optimal harvesting rates $c_{\delta_{i}}(t, x)$ satisfies the inequality

(ii) $c_{\delta_{1}}(t, x) \leq c_{\delta_{2}}(t, x) ;(t, x) \in[0, T] \times D$.

\section{References}

[1] Aase, K., Øksendal, B., Privault, N., Ubøe, J.: White noise generalizations of the Clark-Haussmann-Ocone theorem, with application to mathematical finance. Finance and Stochastics 4 (2000), 465-496.

[2] Chojnowska-Michalik A., Representation theorem for general stochastic delay equations, Bull. Acad. Polon. Sci. Ser. Sci. Math. Astronom. Phys., 26, 7, pp. 635-642, 1978.

[3] Da Prato G., Zabczyk J., Stochastic Equations in Infinite Dimensions, Encyclopedia of Mathematics and its Applications, Cambridge University Press, Cambridge (UK), 1992.

[4] David D., Optimal control of stochastic delayed systems with jumps, preprint, 2008. 
[5] Diekmann O., Van Gils S.A., Verduyn Lunel S.M., Walther H.O., Delay Equations. Functional-, Complex-, and Nonlinear Analysis, SpringerVerlag, New York, 1995.

[6] El Karoui, N., Hamadène, S., BSDEs and risk-sensitive control, zerosum and nonzero-sum game problems of stochastic functional differential equations, Stochastic Processes and their Applications, 107, pp 145-169, 2003.

[7] Elsanousi I., Øksendal B., Sulem A., Some solvable stochastic control problems with delay, Stochastics and Stochastics Reports, 71, pp 69-89, 2000.

[8] Federico S., A stochastic control problem with delay arising in a pension fund model, to appear in Finance and Stochastics, 2009.

[9] Gozzi F., Marinelli C., Stochastic optimal control of delay equations arising in advertising models, Da Prato (ed.) et al., Stochastic partial differential equations and applications VII - Papers of the 7th meeting, Levico Terme, Italy, January 5-10, 2004. Boca Raton, FL: Chapman \& Hall/CRC. Lecture Notes in Pure and Applied Mathematics, 245, pp. 133-148, 2004.

[10] Gozzi F., Marinelli C., Savin S., On controlled linear diffusions with delay in a model of optimal advertising under uncertainty with memory effects, Journal of Optimization, Theory and Applications, to appear.

[11] Kolmanovski, V.B., Shaikhet, L.E. Control of Systems with Aftereffect, American Mathematical Society 1996.

[12] Ikeda, N., Watanabe, S., Stochastic Differential Equations and Diffusion Processes, Second Edition, North-Holland/Kodansha 1989.

[13] Larssen B., Dynamic programming in stochastic control of systems with delay, Stochastics and Stochastics Reports, 74, 3-4, pp 651-673, 2002.

[14] Larssen B., Risebro N.H., When are HJB-equations for control problems with stochastic delay equations finite-dimensional?, Stochastic Analysis and Applications, 21, 3, pp. 643-671, 2003. 
[15] Øksendal B., Sulem A., A maximum principle for optimal control of stochastic systems with delay with applications to finance, Optimal Control and PDE, Essays in Honour of Alain Bensoussan, eds J.L. Menaldi, E. Rofman and A. Sulem, IOS Press, Amsterdam, pp. 64-79, 2001.

[16] Øksendal B., Sulem A., Applied Stochastic Control of Jump Diffusions. Second Edition, Springer, 2007.

[17] Øksendal B., Sulem A. and Zhang T., Optimal control of stochastic delay equations and time-advanced backward stochastic differential equations.Advances in Applied Probability 43:2, 2011.

[18] Øksendal B., Sulem A. and Zhang T., Singular control of SPDEs and time-advanced backward stochastic partial differential equations. Preprint, Manchester-Oslo.

[19] Øksendal B., Proske F. and Zhang T., Backward stochastic partial differential equations with jumps. Stochastics 77 (2005), 381-399.

[20] Øksendal B., Zhang T., Optimal control with partial information for stochastic Volterra equations, International Journal of Stochastic Analysis (to appear).

[21] Pardoux E. and Peng S., Adapted solution of a backward stochastic differential equation, Systems Control Letter 14, 55-61, 1990.

[22] Peng, S., Yang, Z., Anticipated backward stochastic differential equations, The Annals of Probability, 37,3, pp. 877-902, 2009.

[23] Peszat S., Zabczyk J., Stochastic Partial Differential Equations with Lévy Noise, Encyclopedia of Mathematics and its Applications, Vol. 113, Cambridge University Press, Cambridge (UK), 2008.

[24] Protter P.E., Stochastic Integration and Differential Equations, 2nd edition, Springer-Verlag, Berlin-Heidelberg-New York, 2003.

[25] Reiss M., Riedle M., Van Gaans O., Delay differential equations driven by Lévy processes: stationarity and Feller's properties, Stochastic Processes and their Applications, Vol.116, pp. 1409-1432, 2006. 
[26] Situ R., On solutions of backward stochastic differential equations with jumps and applications, Stochastic Processes and their Applications 66, 209-236, 1997. 